\title{
MicroRNA-612 is downregulated by platelet-derived growth factor-BB treatment and has inhibitory effects on vascular smooth muscle cell proliferation and migration via directly targeting AKT2
}

\author{
CHEN CHEN $^{1}$, YAN YAN ${ }^{2}$ and XIAODAN LIU ${ }^{3}$ \\ ${ }^{1}$ Department of Cardiology, Affiliated Hospital of Binzhou Medical College, Binzhou, Shandong 256603; \\ ${ }^{2}$ Emergency Centre, Beijing Anzhen Hospital, Capital Medical University, Beijing 100029; ${ }^{3}$ Department of \\ Hematology, Affiliated Hospital of Binzhou Medical College, Binzhou, Shandong 256603, P.R. China
}

Received November 4, 2016; Accepted April 21, 2017

DOI: $10.3892 /$ etm.2017.5428

\begin{abstract}
Abnormal proliferation and migration of vascular smooth muscle cells (VSMCs) has been implicated in neointimal formation, and therefore is suggested to contribute to arteriosclerosis and restenosis. Previous studies have suggested that some microRNAs (miRs) serve crucial roles in VSMC proliferation and invasion; however, the underlying mechanism remains largely unknown. In the present study, it was demonstrated that treatment with platelet-derived growth factor (PDGF)-BB significantly promoted the proliferation and migration of VSMCs, and decreased miR-612 levels in VSMCs. Overexpression of miR-612 significantly inhibited PDGF-BB-induced migration and invasion of VSMCs, through inducing cell cycle arrest at G1 stage. AKT2 was further identified as a direct target gene of miR-612, and its expression was negatively regulated by miR-612 in VSMCs. Further investigation confirmed that overexpression of miR-612 suppressed the PDGF-BB-induced upregulation of AKT2 protein expression. In conclusion, the present study demonstrated that miR-612 is downregulated by PDGF-BB treatment and has inhibitory effects on VSMC proliferation and migration via targeting AKT2. These findings suggest that miR-612 may be used as a potential therapeutic candidate for neointimal formation in patients with atherosclerosis.
\end{abstract}

\section{Introduction}

During the repair of vascular injury, various cytokines, such as platelet-derived growth factor (PDGF), that are able to

Correspondence to: Dr Xiaodan Liu, Department of Hematology, Affiliated Hospital of Binzhou Medical College, 661 Huanghe 2 Road, Binzhou, Shandong 256603, P.R. China

E-mail: dr_liuxiaodan@qq.com

Key words: vascular smooth muscle cells, microRNA, proliferation, migration, AKT2 stimulate the proliferation and migration of vascular smooth muscle cells (VSMCs) are released (1). Unbalanced proliferation and migration of VSMCs has been demonstrated to serve key roles in neointimal formation and thus are important for arteriosclerosis and restenosis following coronary intervention or vein grafting (2). Therefore, exploring the underlying molecular mechanism may be beneficial for the development of effective strategies for inhibiting this process, and thus reducing the incidence of cardiovascular diseases $(3,4)$.

microRNAs (miRs), a type of non-coding RNAs that are 18-25 nucleotides in length, are key regulators for gene expression through binding to the 3'-untranslated region (UTR) of target mRNAs, causing mRNA degradation or translation inhibition $(5,6)$. Through inhibiting the protein expression of their target genes, miRs serve important roles in various cellular biological processes, including cell survival, differentiation, proliferation, apoptosis, migration, angiogenesis and tumorigenesis (7-9). Recently, miRs have been found to participate in the development and progression of atherosclerosis and restenosis, and alterations of $\mathrm{miR}$ expression profiles have been identified in these vascular diseases $(10,11)$. For instance, in arterial lesions following balloon injury, many miRs have been found to be significantly upregulated or downregulated (11). Furthermore, some miRs have been reported to have promoting or suppressive roles in abnormal proliferation and migration of VSMCs (2). For instance, Liu et al (12) reported that miR-221 and miR-222 were necessary for the proliferation of VSMCs and neointimal hyperplasia, and inhibition of miR-221 and miR-222 expression in rat carotid arteries reduced VSMC proliferation and suppressed neointimal formation following angioplasty. Sun et al (13) demonstrated that miR-146a serves a promoting role in VSMC proliferation in vitro and vascular neointimal hyperplasia in vivo, at least in part, by directly targeting Kruppel-like factor 4. Recently, miR-612 has been demonstrated to have suppressive effects on the stemness of liver cancer, as well as tumor growth and metastasis in colorectal cancer $(14,15)$. However, the molecular mechanism of miR-612 on vascular cell dynamics remains to be elucidated. 
The aim of the present study was to investigate the exact role of miR-612 in platelet-derived growth factor (PDGF)-BB-induced proliferation and migration of VSMCs, as well as the underlying mechanism.

\section{Materials and methods}

Animals. The present study was approved by the Animal Care and Use Committee of the Affiliated Hospital of Binzhou Medical College (Binzhou, China). Animal experiments were consistent with the National Institutes of Health Guide for the Care and Use of Laboratory Animals. A total of 5 male Sprague Dawley rats (200-250 g, 8 weeks old) were purchased from the Shandong Laboratory Animal Center (Jinan, China) and housed in light-controlled (12 h light/dark cycle) and temperature-controlled $\left(22 \pm 2^{\circ} \mathrm{C}\right)$ room with free access to food and water.

Cell culture and treatment. The present study was approved by the Ethics Committee of the Affiliated Hospital of Binzhou Medical College (Binzhou, China). VSMCs were isolated from the thoracic aortas of rats, and cultured in Dulbecco's modified Eagle's medium (DMEM; Thermo Fisher Scientific, Inc., Waltham, MA, USA) supplemented with $10 \%$ fetal bovine serum (FBS; Thermo Fisher Scientific, Inc.), $10 \mathrm{mmol} / 1$ 4-(2-hydroxyethyl)-1-piperazineethanesulphonic acid (Sigma-Aldrich; Merck KGaA, Darmstadt, Germany), $100 \mathrm{U} / \mathrm{ml}$ penicillin (Sigma-Aldrich; Merck KGaA) and $100 \mathrm{mg} / \mathrm{ml}$ streptomycin (Sigma-Aldrich; Merck KGaA) at $37^{\circ} \mathrm{C}$ in a humidified atmosphere containing $5 \% \mathrm{CO}_{2}$ and $95 \%$ air. VSMCs of fifth passage were used. VSMCs without any treatment were used as the control group. VSMCs in the PDGF-BB group were cultured to $70 \%$ confluence, and subsequently incubated at $37^{\circ} \mathrm{C}$ with PDGF-BB $(20 \mathrm{ng} / \mathrm{ml}$; Thermo Fisher Scientific, Inc.) for $6 \mathrm{~h}$.

Reverse transcription-quantitative polymerase chain reaction (RT-qPCR). TRIzol reagent (Thermo Fisher Scientific, Inc.) was used to extract total RNA from VSMCs, which was to produce cDNA using a PrimeScript RT reagent kit (Takara Biotechnology Co., Ltd., Dalian, China), according to the manufacturer's protocol. qPCR was conducted using a mirVana miRNA Detection kit (Thermo Fisher Scientific, Inc.) with an ABI 7500 thermocycler (Thermo Fisher Scientific, Inc.). The sequences for miR-612 (cat. no. HmiR0173) and the reference gene U6 (cat. no. HmiRQP9001) were not supplied by the manufacturer (Fulengen, Guangzhou, China). The reaction conditions for PCR were as follows: Pre-degeneration at $95^{\circ} \mathrm{C}$ for $3 \mathrm{~min}$ and 40 cycles of $95^{\circ} \mathrm{C}$ for $30 \mathrm{sec}, 60^{\circ} \mathrm{C}$ for $30 \mathrm{sec}$. Independent experiments were repeated three times. The relative expressions of mRNA were analyzed by use of the $2^{-\Delta \Delta \mathrm{Cq}}$ method (16).

Cell transfection. Lipofectamine ${ }^{\circledR} 2000$ (Thermo Fisher Scientific, Inc.) was used to perform cell transfection, in accordance with the manufacturer's protocol. Briefly, VSMCs were cultured to $70 \%$ confluence, and resuspended in serum-free DMEM. Scramble miR mimic (miR-NC) or miR-612 mimic (100 nM, Yearthbio, Changsha, China) were diluted in OPTI-MEM (Thermo Fisher Scientific, Inc.), respectively, which was then added with diluted Lipofectamine ${ }^{\circledR} 2000$. The sequences for scramble miR mimic (cat. no. AM1011) and miR-612 (cat. no. AM1423) mimic were not supplied by the manufacturer (Fulengen). Following incubation for $20 \mathrm{~min}$ at room temperature, the mixture was added into the cell suspension. Following incubation at $37^{\circ} \mathrm{C}$ and $5 \% \mathrm{CO}_{2}$ for $6 \mathrm{~h}$, the transfection mixture was replaced with DMEM with $10 \%$ FBS. At $48 \mathrm{~h}$ following transfection, the expression levels of miR-612 were examined using RT-qPCR following the procedure as above. Following transfection, VSMCs were treated with PDGF-BB for $6 \mathrm{~h}$.

MTT assay. VSMCs $\left(5 \times 10^{3}\right)$ in each group were seeded in a 96-well plate, and $100 \mu \mathrm{l}$ fresh serum-free DMEM with $0.5 \mathrm{~g} / \mathrm{l}$ MTT was added to each well. Following incubation at $37^{\circ} \mathrm{C}$ for 0, 24, 48 and $72 \mathrm{~h}$, the medium containing MTT was removed, and $100 \mu \mathrm{l}$ dimethyl sulfoxide was added. Following incubation at $37^{\circ} \mathrm{C}$ for $10 \mathrm{~min}$, the absorbance at $570 \mathrm{~nm}$ of each sample was measured using a plate reader (Infinite M200; Tecan Group, Ltd., Männedorf, Switzerland).

Transwell assay. Transwell assay was conducted to examine cell migration using a 24-well Transwell chamber (Chemicon; EMD Millipore, Billerica, MA USA). VSMCs cell suspension (containing $5 \times 10^{5}$ cells) was added to the upper chamber, and DMEM containing 10\% FBS was added into the lower chamber. Following incubation in a $37^{\circ} \mathrm{C}$ humidified atmosphere at $5 \% \mathrm{CO}_{2}$ for $24 \mathrm{~h}$, cells on the interior of the inserts were removed using a cotton-tipped swab. Migratory cells on the lower surface of the membrane were stained at room temperature for $10 \mathrm{~min}$ with gentian violet (Sigma-Aldrich; Merck $\mathrm{KGaA}$ ), rinsed with water at room temperature for $10 \mathrm{~min}$, dried in air at room temperature for $20 \mathrm{~min}$, and counted under a light microscope (magnification, x200; Nikon Corporation, Tokyo, USA).

Cell cycle analysis. VSMCs $\left(5 \times 10^{6}\right)$ in each group were fixed in $70 \%$ ethanol overnight at $-20^{\circ} \mathrm{C}$, and centrifuged at $4{ }^{\circ} \mathrm{C}$ at $1,000 \times \mathrm{g}$ for $5 \mathrm{~min}$, washed in PBS, and then centrifuged at $4^{\circ} \mathrm{C}$ at $1,000 \mathrm{x}$ g for $5 \mathrm{~min}$. Cells were resuspended in $300 \mu \mathrm{l}$ propidium iodide staining buffer (Beyotime Institute of Biotechnology, Haimen, China) and incubated for $30 \mathrm{~min}$ at room temperature. DNA content analyses were performed using a flow cytometer (C6; BD Biosciences, Franklin Lakes, NJ, USA).

Western blot analysis. Cold RIPA lysis buffer (Beyotime Institute of Biotechnology) was used to solubilize VSMCs. The concentration of protein was determined with a BCA Protein Assay kit (Thermo Fisher Scientific, Inc.). Protein $(50 \mu \mathrm{g})$ was separated by $10 \%$ SDS-PAGE, and then transferred to a polyvinylidene fluoride membrane (Thermo Fisher Scientific, Inc.). The membrane was blocked at room temperature in 5\% non-fat dried milk in TBST (Thermo Fisher Scientific, Inc.) for $2 \mathrm{~h}$. Following three 5 min washes with TBST, the membrane was incubated with rabbit anti-total AKT2 (1:100; ab175354), rabbit anti-phosphorylated AKT2 $(1: 100 ;$ ab38513) or rabbit anti-GAPDH antibody $(1: 100$; ab9485; all Abcam, Cambridge, UK) at $4^{\circ} \mathrm{C}$ overnight. Following three $5 \mathrm{~min}$ washes with TBST, the membrane 
A

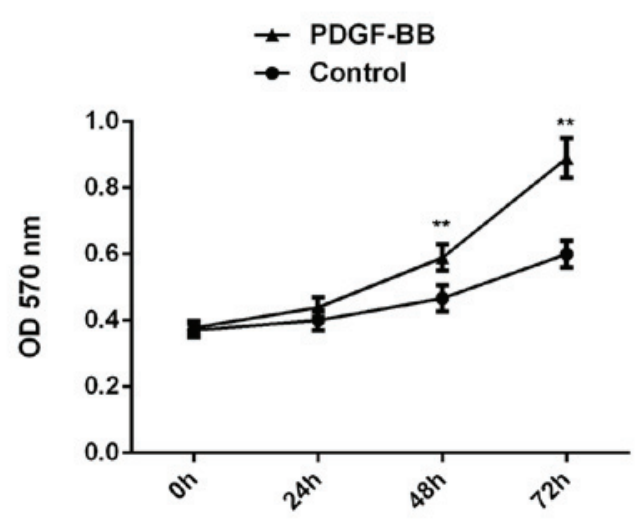

B
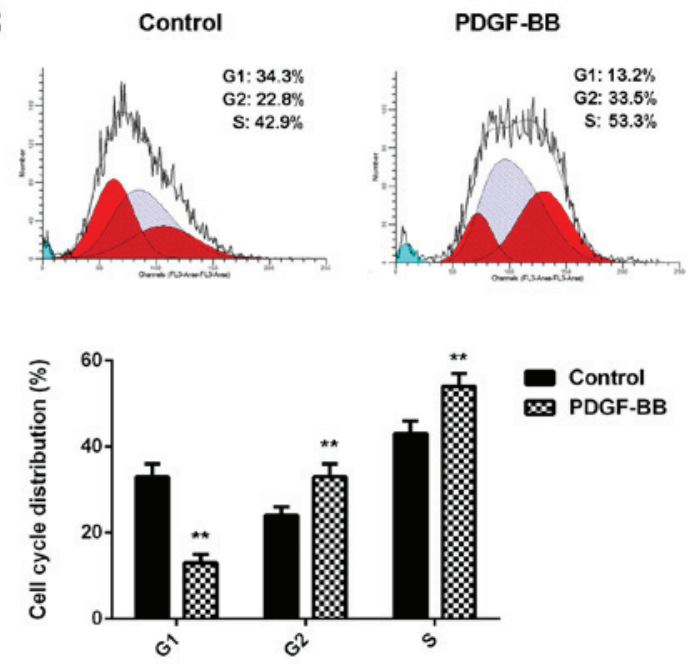

C

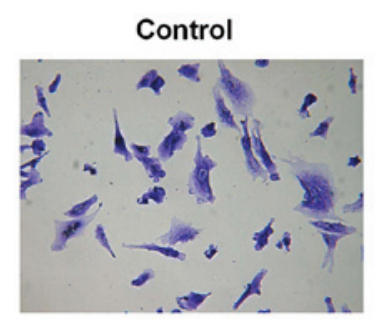

PDGF-BB

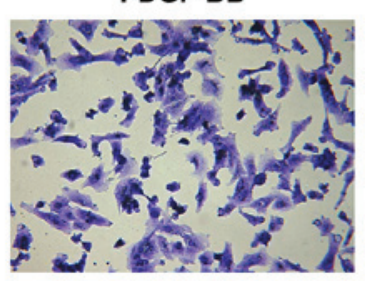

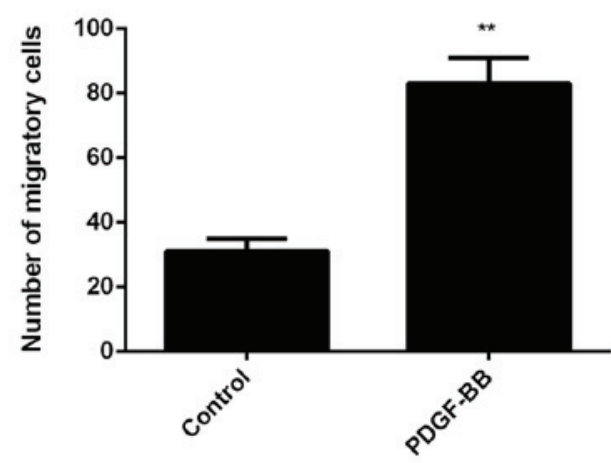

Figure 1. Vascular smooth muscle cells were treated with PDGF-BB for 6 h. (A) An MTT assay was conducted to examine cell proliferation. (B) Flow cytometry was conducted to examine cell cycle distribution. (C) Transwell assay was used to examine cell migration. ${ }^{* *} \mathrm{P}<0.01$ vs. control. PDGF-BB, platelet-derived growth factor-BB; OD, optical density.

was incubated with horseradish peroxidase conjugated goat anti-rabbit secondary antibody (1:5,000; ab7090; Abcam) for $40 \mathrm{~min}$ at room temperature. Following three $5 \mathrm{~min}$ washes with TBST, the immune complexes on PVDF membrane were then detected using an enhanced chemiluminescent western blotting kit (Thermo Fisher Scientific, Inc.), according to the manufacturer's protocol. The relative protein expression was analyzed using ImageJ software 1.4 (National Institutes of Health, Bethesda, MD, USA), and represented as the density ratio vs. GAPDH.

Bioinformatics analysis and dual-luciferase reporter assay. TargetScan Human 3.1 (www.targetscan.org) was used to predict the potential target of miR-216. The mutant type (MT) of AKT2 3'UTR was constructed using an Easy Mutagenesis System kit (Promega Corporation, Madison, WI, USA), in accordance with the manufacturer's protocol. The wild-type (WT) or MT of AKT2 3'UTR was then inserted into the pMIR-REPORT miR Expression Reporter vector (Promega Corporation). VSMCs in the control group were co-transfected with WT-AKT2-3'UTR or MT-AKT2-3'UTR plasmid, and miR-NC or miR-216 mimic, using Lipofectamine ${ }^{\circledR} 2000$, respectively. At $48 \mathrm{~h}$ following transfection, the Dual-Luciferase Reporter Assay System (Promega Corporation) was used to determine the luciferase activity, and the Renilla luciferase activity was normalized to the firefly luciferase activity.
Statistical analysis. Data are presented as the mean \pm standard deviation. Statistical analysis was performed using SPSS 20 (IBM Corp., Armonk, NY, USA). The differences between two groups were analyzed using Student's t-test. $\mathrm{P}<0.05$ was considered to indicate a significant difference.

\section{Results}

Treatment with PDGF-BB promoted the proliferation and migration of VSMCs. In the present study, VSMCs in PDGF-BB group were treated with PDGF-BB for $6 \mathrm{~h}$. VSCMs without any treatment were used as the control group. Following treatment, the proliferation of VSMCs was evaluated. As shown in Fig. 1A, the proliferation of VSMCs was significantly increased in the PDGF-BB group compared with the control group at 48 and $72 \mathrm{~h}$. Flow cytometry revealed that the percentage of VSMCs at G1 stage was significantly lower in the PDGF-BB group compared with the control group, which suggested that treatment with PDGF-BB is able to promote cell cycle progression (Fig. 1B). Cell migration in each group was subsequently evaluated, and it was indicated that the migration of VSMCs was significantly upregulated in the PDGF-BB group when compared with the control group (Fig. 1C). As such, these findings indicated that treatment with PDGF-BB promoted the proliferation and migration of VSMCs. 

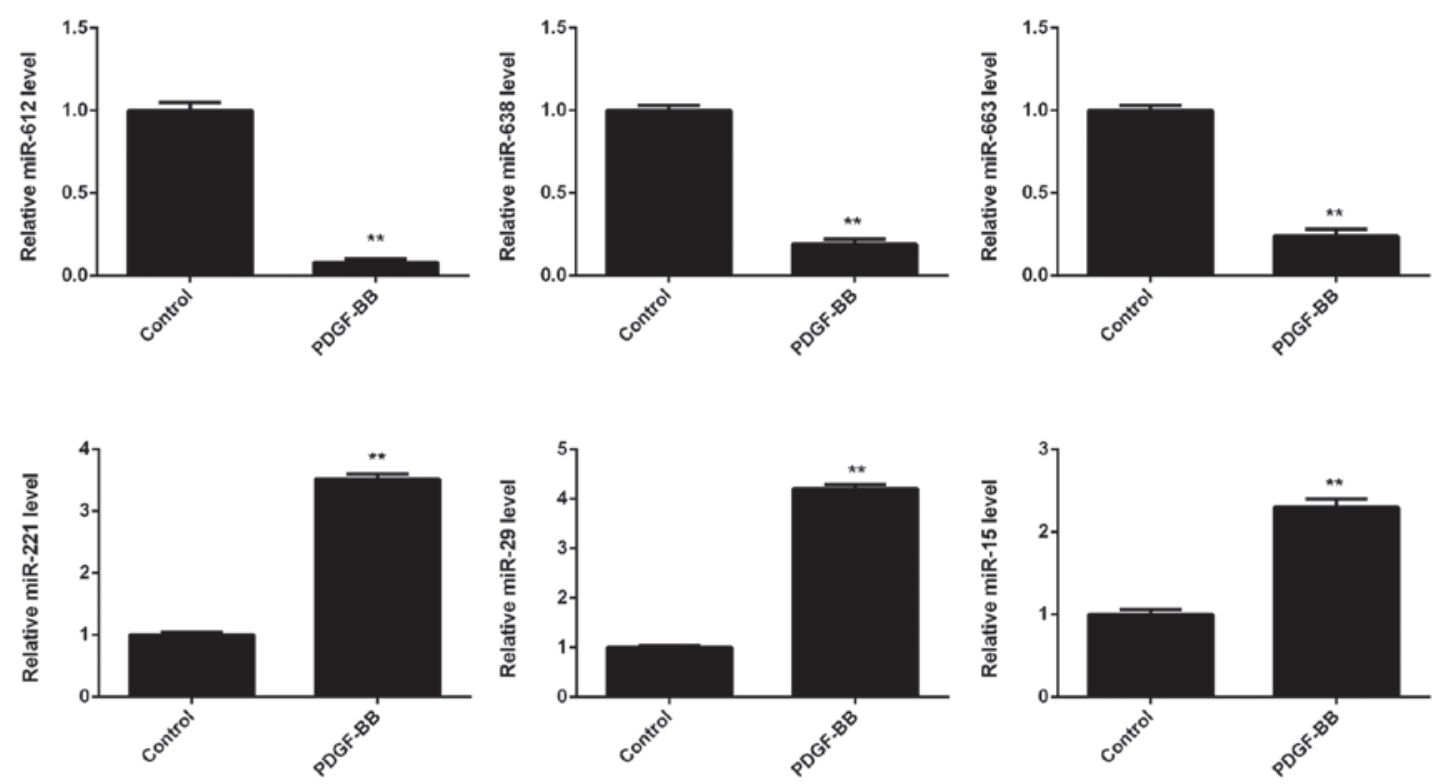

Figure 2. Vascular smooth muscle cells were treated with PDGF-BB for $6 \mathrm{~h}$. Reverse transcription-quantitative polymerase chain reaction was subsequently conducted to examine the expression of various miRs. ${ }^{* *} \mathrm{P}<0.01$ vs. control. PDGF-BB, platelet-derived growth factor-BB; miR, microRNA.

Treatment with PDGF-BB downregulated miR-612 expression in VSMCs. The expression of several miRs in VSMCs was subsequently evaluated, with or without PDGF-BB treatment. As shown in Fig. 2, miR-612, miR-638, and miR-663 were significantly downregulated in the PDGF-BB group compared with controls, whereas miR-221, miR-29, and miR-15 were significantly upregulated. Furthermore, miR-612 demonstrated the greatest downregulation in VSMCs treated with PDGF-BB, when compared with the control group (Fig. 2).

Overexpression of miR-612 attenuated the proliferation and migration of VSMCs induced by PDGF-BB treatment. The regulatory effects of miR-612 on the proliferation and migration of VSMCs induced by PDGF-BB treatment were then evaluated. VSMCs were transfected with miR-612 mimic or miR-NC mimic and after transfection the miR-612 levels were significantly increased in the miR-612 group compared with the miR-NC group (Fig. 3A). VSMCs in each group were then treated with PDGF-BB for $6 \mathrm{~h}$. MTT assay data indicated that the proliferation of VSMCs was significantly reduced in miR-612 group compared with the miR-NC group at 48 and $72 \mathrm{~h}$ (Fig. 3B). Flow cytometry data indicated that the cell percentage in the G1 stage was significantly higher in the miR-612 group compared with the miR-NC group, suggesting that overexpression of miR-612 led to a significant cell cycle arrest at G1 stage, which partially contributes to decreased VSMC proliferation (Fig. 3C). Further investigation revealed that the migration of VSMCs was also significantly reduced in the miR-612 group compared with the miR-NC group (Fig. 3D). Therefore, overexpression of miR-612 attenuated the proliferation and migration of VSMCs induced by PDGF-BB treatment.

AKT2 is a direct target gene of miR-612 in VSMCs. As miRs function through regulation of their target genes, the potential target gene of miR-612 in VSMCs was investigated. Data from the TargetScan Human 3.1 database indicated that AKT2 was a putative target gene of miR-612. To confirm this prediction, WT- and MT-AKT2-3'UTR luciferase reporter plasmids were constructed (Fig. 4A and B), and a luciferase reporter gene assay was performed using VSMCs. As shown in Fig. 4C, the luciferase activity was significantly downregulated in VSMCs transfected with WT-AKT2-3'UTR luciferase reporter plasmid and miR-612 mimic, when compared with the control group; however, this was ameliorated by transfection with MT-AKT2-3'UTR luciferase reporter plasmid. These findings indicate that miR-612 directly binds to the 3'UTR of AKT2 mRNA in VSMCs.

The effects of miR-612 on the protein expression of AKT2 in VSMCs were subsequently evaluated. As shown in Fig. 4D, overexpression of miR-612 significantly reduced the protein levels of AKT2 in VSMCs. To further confirm these findings, VSMCs were transfected with miR-612 inhibitor, and qPCR data indicated that the miR-612 levels were significantly decreased in the miR-612 inhibitor group, when compared with those in the NC inhibitor group (Fig. 4E). Western blot data then showed that knockdown of miR-612 significantly increased the protein expression of AKT2 in VSMCs (Fig. 4F). Together, these data suggest that miR-612 negatively regulates the protein expression of its target AKT2 in VSMCs.

Overexpression of miR-612 inhibited the PDGF-BB-induced upregulation of AKT2 in VSMCs. Finally, the molecular mechanism of miR-612 in PDGF-BB-treated VSMCs was evaluated. As shown in Fig. 5A, treatment with PDGF-BB significantly upregulated the protein expression of total AKT2 and phosphorylated AKT2 in VSMCs, when compared with the control group. However, overexpression of miR-612 significantly downregulated the protein expression of t-AKT2 and p-AKT2 in VSMCs treated with PDGF-BB, when compared with the miR-NC group (Fig. 5B). These findings suggest that miR-612 has inhibitory effects on the PDGF-BB-induced upregulation of AKT signaling in VSMCs. 
A

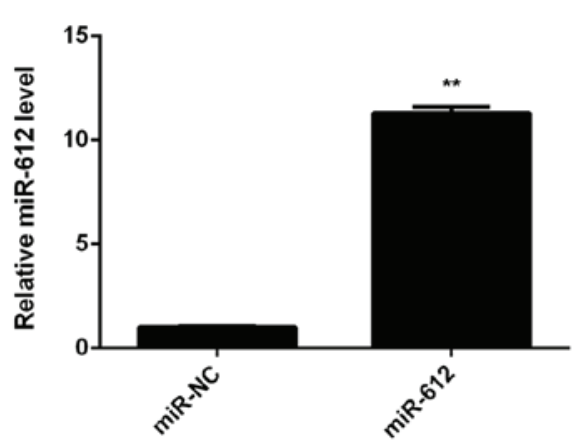

C
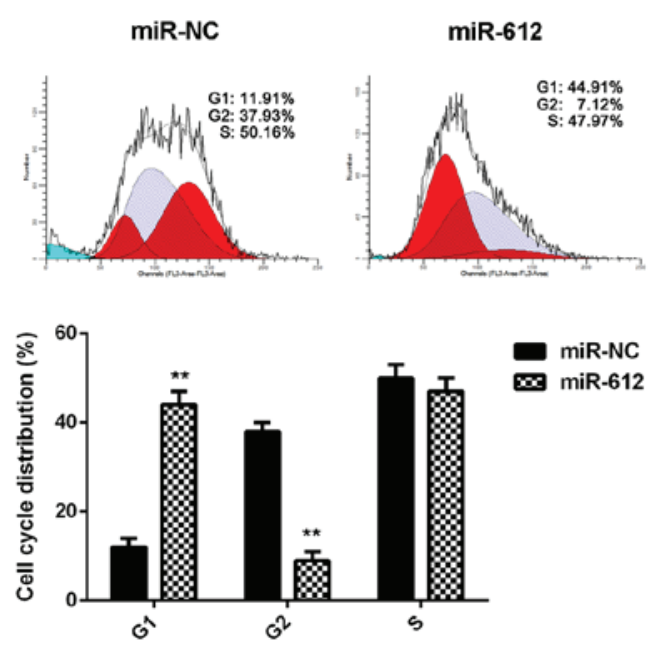

B

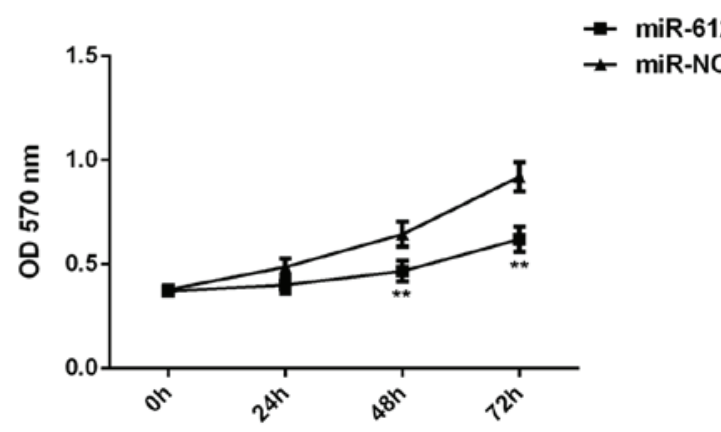

D
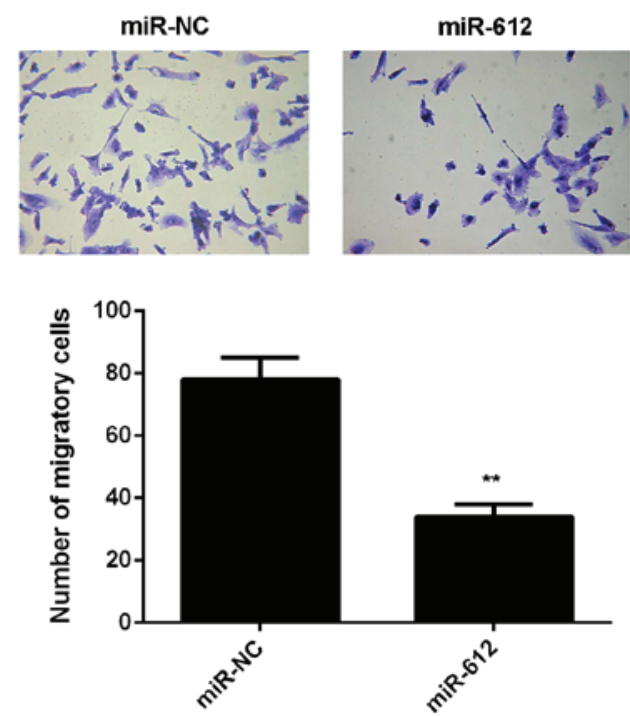

Figure 3. VSMCs were transfected with miR-612 mimic or miR-NC. (A) Reverse transcription-quantitative polymerase chain reaction was conducted to examine miR-612 levels. Subsequently, VSMCs in each group were treated with PDGF-BB for $6 \mathrm{~h}$. (B) MTT assay was conducted to examine cell proliferation. (C) Flow cytometry was conducted to examine cell cycle distribution. (D) Transwell assay was used to examine cell migration. ${ }^{* *} \mathrm{P}<0.01 \mathrm{vs}$. miR-NC. VSMC, vascular smooth muscle cell; miR, microRNA; miR-NC, scramble miR mimic; PDGF-BB, platelet-derived growth factor-BB; OD, optical density.

\section{Discussion}

Various miRs have been suggested to serve promoting or suppressive roles in neointimal formation (17,18); however, the underlying mechanism remains unclear. In the present study, it was demonstrated that treatment with PDGF-BB significantly promoted the proliferation and migration of VSMCs, and decreased the miR-612 levels in VSMCs. Overexpression of miR-612 significantly inhibited PDGF-BB-induced migration and invasion of VSMCs, through inducing a cell cycle arrest at G1 stage. AKT2 was further identified as a direct target gene of miR-612, and its expression was negatively regulated by miR-612 in VSMCs. Furthermore, overexpression of miR-612 suppressed the PDGF-BB-induced upregulation of AKT signaling.

The proliferation and migration of VSMCs have promoting effects on the development of arteriosclerosis and restenosis, and PDGF has been demonstrated to stimulate VSMC proliferation and migration through multiple mechanisms, including the modulation of miR expression (19-21). For example, miR-15b expression, which is induced by PDGF signaling, is required for the proliferation of VSMCs (19). In the present study, treatment with PDGF-BB induced a significant reduction in the expression levels of several miRs in VSMCs, accompanied by increased cell proliferation and migration. It was hypothesized that these downregulated miRs may have a role in VSMC proliferation and migration. As miR-612 exhibited the greatest downregulation, VSMCs were transfected with miR-612 mimic to upregulate its expression. Ectopic expression of miR-612 was found to significantly attenuate the proliferation and migration of VSMCs induced by PDGF-BB treatment, which confirmed the hypothesis.

As miRs function through regulating the expression of their target genes (9), potential targets of miR-612 were evaluated and AKT2 was identified as a direct target of miR-612 in VSMCs via luciferase reporter gene assay. AKT2 encodes AKT serine/threonine kinase 2, which is an important member of PI3K/AKT signaling and capable of phosphorylating several downstream proteins such as p70S6 kinase, mechanistic target of rapamycin and glycogen synthase kinase $3(22,23)$. A previous study confirmed that AKT2 has a promoting role in VSMC migration and neointimal formation (24). Furthermore, AKT2 is associated with CXCL16-triggered platelet activation and adhesion, and thus may be associated with vascular inflammation and thrombo-occlusive diseases (25). Rotllan et al also reported that hematopoietic AKT2 deficiency attenuated the progression of atherosclerosis (26). In the present study, miR-612 was demonstrated to negatively regulate the protein expression 
A

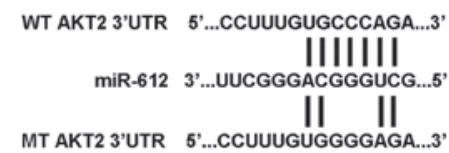

C



E



B


D
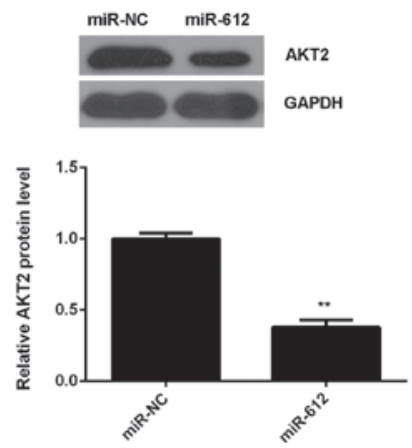

$F$

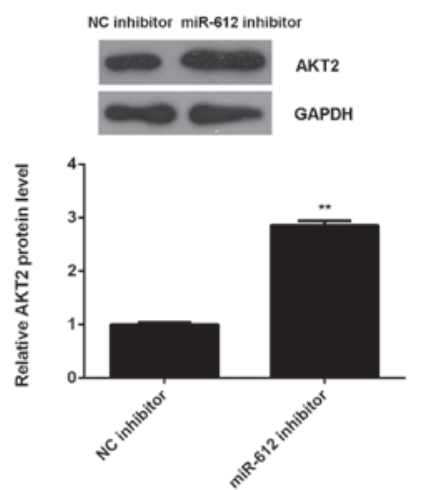

Figure 4. (A and B) WT- and MT-AKT2-3'UTR luciferase reporter plasmids were generated. (C) Luciferase reporter gene assay was performed to determine luciferase activity. ${ }^{* *} \mathrm{P}<0.01$ vs. control. (D) Western blotting was used to examine the protein expression of AKT2 in VSMCs transfected with miR-612 mimic or miR-NC. ${ }^{* *} \mathrm{P}<0.01$ vs. miR-NC. VSMCs were transfected with NC inhibitor or miR-612 inhibitor, respectively. (E) Reverse transcription-quantitative polymerase chain reaction was conducted to evaluate miR-612 levels, and (F) western blotting was used to examine the protein expression of AKT2. ${ }^{* *} \mathrm{P}<0.01 \mathrm{vs}$. NC inhibitor. WT, wild-type; MT, mutant type; UTR, untranslated region; VSMC, vascular smooth muscle cell; miR, microRNA; miR-NC, scramble miR mimic.

A
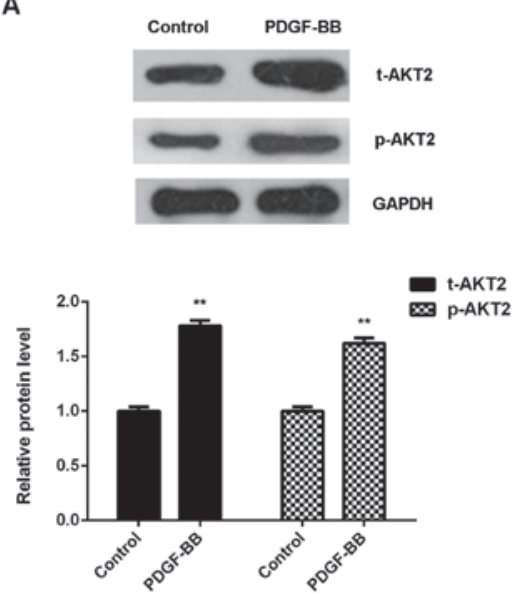

B
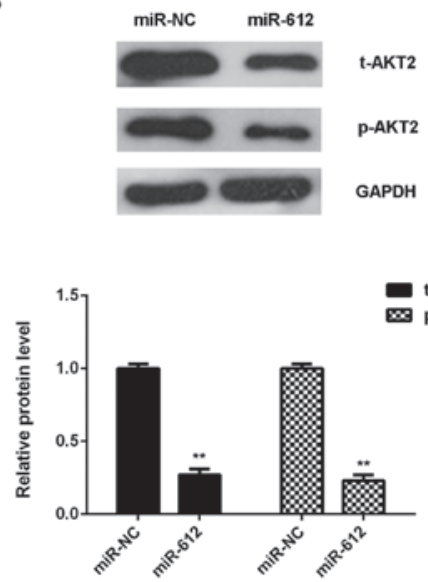

Figure 5. VSMCs were treated with PDGF-BB for $6 \mathrm{~h}$. (A) Western blotting was used to examine the protein expression of t-AKT2 and p-AKT2. ${ }^{* *} \mathrm{P}<0.01$ vs. control. (B) VSMCs were transfected with miR-612 mimic or miR-NC and treated with PDGF-BB for $6 \mathrm{~h}$, and western blotting was used to examine the protein expression of t-AKT2 and p-AKT2. ${ }^{* *} \mathrm{P}<0.01$ vs. miR-NC. VSMC, vascular smooth muscle cell; PDGF-BB, platelet-derived growth factor-BB; t-, total; p-, phosphorylated; miR, microRNA; miR-NC, scramble miR mimic.

of AKT2 and to inhibit the PDGF-BB-induced upregulation of AKT2 protein expression in VSMCs. These findings suggest that miR-612 is able to inhibit the PDGF-BB-induced VSMC proliferation and invasion through inhibition of AKT2 signaling.
In addition to miR-612, various other miRs have also been found to serve a suppressive role in VSMC proliferation and/or migration. For instance, miR-21 inhibits PDGF-induced proliferation and migration of human aortic VSMCs through 
inhibiting the expression of activator protein-1 (27). miR-638, which is highly expressed in VSMCs, is able to inhibit PDGF-BB-induced cell proliferation and migration through directly targeting neuron-derived orphan receptor 1 (28). miR-24 inhibits high glucose-induced VSMC proliferation and migration by targeting high mobility group box 1 protein (29). Furthermore, the targeting association between miR-612 and AKT2 has also previously been found in colon and liver cancers $(15,30)$. For instance, Sheng et al (15) recently reported that miR-612 is able to inhibit colorectal cancer growth and metastasis by targeting AKT2. Accordingly, the present study expands the understanding of miR-612 functions.

In conclusion, the present study is, to the best of our knowledge the first study to demonstrate that miR-612 has suppressive effects on PDGF-BB-induced proliferation and migration of VSMCs, at least in part, by directly targeting AKT2. These findings suggest that miR-612 may be a potential candidate for inhibiting neointimal formation and thus preventing arteriosclerosis or restenosis following coronary intervention or vein grafting.

\section{References}

1. Yi N, Chen SY, Ma A, Chen PS, Yao B, Liang TM and Liu C: Tunicamycin inhibits PDGF-BB-induced proliferation and migration of vascular smooth muscle cells through induction of HO-1. Anat Rec (Hoboken) 295: 1462-1472, 2012.

2. Zhang C: MicroRNA-145 in vascular smooth muscle cell biology: A new therapeutic target for vascular disease. Cell Cycle 8 3469-3473, 2009.

3. Obata JE, Nakamura T, Kitta Y, Saito Y, Sano K, Fujioka D, Kawabata K and Kugiyama K: In-stent restenosis is inhibited in a bare metal stent implanted distal to a sirolimus-eluting stent to treat a long de novo coronary lesion with small distal vessel diameter. Catheter Cardiovasc Interv 82: E777-E787, 2013.

4. Mazurova VV, Sukhorukov OE and Zakharova OV: Comparing moderately late results of the application of stents coated with a medicinal antiproliferative agent for the treatment of patients with various forms of coronary heart disease: Their efficacy and safety. Klin Med (Mosk) 90: 30-35, 2012 (In Russian).

5. Moss EG: MicroRNAs: Hidden in the genome. Curr Biol 12: R138-R140, 2002.

6. Bartel DP: MicroRNAs: Genomics, biogenesis, mechanism, and function. Cell 116: 281-297, 2004.

7. Zhou W, Zou B, Liu L, Cui K, Gao J, Yuan S and Cong N: MicroRNA-98 acts as a tumor suppressor in hepatocellular carcinoma via targeting SALL4. Oncotarget 7: 74059-74073, 2016.

8. Yao J, Deng B, Zheng L, Dou L, Guo Y and Guo K: miR-27b is upregulated in cervical carcinogenesis and promotes cell growth and invasion by regulating CDH11 and epithelial-mesenchymal transition. Oncol Rep 35: 1645-1651, 2016.

9. Ambros V: The functions of animal microRNAs. Nature 431: 350-355, 2004

10. Chen LJ, Lim SH, Yeh YT, Lien SC and Chiu JJ: Roles of microRNAs in atherosclerosis and restenosis. J Biomed Sci 19: 79, 2012.

11. Weber C, Schober A and Zernecke A: MicroRNAs in arterial remodelling, inflammation and atherosclerosis. Curr Drug Targets 11: 950-956, 2010.

12. Liu X, Cheng Y, Zhang S, Lin Y, Yang J and Zhang C: A necessary role of miR-221 and miR-222 in vascular smooth muscle cell proliferation and neointimal hyperplasia. Circ Res 104: 476-487, 2009.

13. Sun SG, Zheng B, Han M, Fang XM, Li HX, Miao SB, Su M, Han Y, Shi HJ and Wen JK: miR-146a and Krüppel-like factor 4 form a feedback loop to participate in vascular smooth muscle cell proliferation. EMBO Rep 12: 56-62, 2011.

14. Tang J, Tao ZH, Wen D, Wan JL, Liu DL, Zhang S, Cui JF, Sun HC, Wang L, Zhou J, et al: MiR-612 suppresses the stemness of liver cancer via Wnt/ $\beta$-catenin signaling. Biochem Biophys Res Commun 447: 210-215, 2014.
15. Sheng $L$, He $P$, Yang $X$, Zhou $M$ and Feng Q: miR-612 negatively regulates colorectal cancer growth and metastasis by targeting AKT2. Cell Death Dis 6: e1808, 2015.

16. Livak KJ and Schmittgen TD: Analysis of relative gene expression data using real-time quantitative PCR and the 2(-Delta Delta C(T)) Method. Methods 25: 402-408, 2001

17. Lee J, Lim S, Song BW, Cha MJ, Ham O, Lee SY, Lee C, Park JH, Bae Y, Seo HH, et al: MicroRNA-29b inhibits migration and proliferation of vascular smooth muscle cells in neointimal formation. J Cell Biochem 116: 598-608, 2015.

18. Li TJ, Chen YL, Gua CJ, Xue SJ, Ma SM and Li XD: MicroRNA $181 \mathrm{~b}$ promotes vascular smooth muscle cells proliferation through activation of PI3K and MAPK pathways. Int J Clin Exp Pathol 8: 10375-10384, 2015.

19. Kim S and Kang H: miR-15b induced by platelet-derived growth factor signaling is required for vascular smooth muscle cell proliferation. BMB Rep 46: 550-554, 2013.

20. Gan J, Li P, Wang Z, Chen J, Liang X, Liu M, Xie W, Yin R and Huang F: Rosuvastatin suppresses platelet-derived growth factor-BB-induced vascular smooth muscle cell proliferation and migration via the MAPK signaling pathway. Exp Ther Med 6: 899-903, 2013.

21. Guan S, Tang Q, Liu W, Zhu R and Li B: Nobiletin Inhibits PDGF-BB-induced vascular smooth muscle cell proliferation and migration and attenuates neointimal hyperplasia in a rat carotid artery injury model. Drug Dev Res 75: 489-496, 2014.

22. Frias A, Lambies G, Viñas-Castells R, Martínez-Guillamon C, Dave N, García de Herreros A and Díaz VM: A switch in Akt isoforms is required for notch-induced Snaill expression and protection from cell death. Mol Cell Biol 36: 923-940, 2015.

23. Shiratsuchi $\mathrm{H}$ and Basson MD: Akt2, but not Akt1 or Akt3 mediates pressure-stimulated serum-opsonized latex bead phagocytosis through activating mTOR and p70 S6 kinase. J Cell Biochem 102: 353-367, 2007.

24. Zhang W, Zhang X, González-Cobos JC, Stolwijk JA, Matrougui $\mathrm{K}$ and Trebak M: Leukotriene-C4 synthase, a critical enzyme in the activation of store-independent Orail/Orai3 channels, is required for neointimal hyperplasia. J Biol Chem 290: 5015-5027, 2015

25. Borst O, Münzer P, Gatidis S, Schmidt EM, Schönberger T, Schmid E, Towhid ST, Stellos K, Seizer P, May AE, et al: The inflammatory chemokine CXC motif ligand 16 triggers platelet activation and adhesion via CXC motif receptor 6-dependent phosphatidylinositide 3-kinase/Akt signaling. Circ Res 111: 1297-1307, 2012.

26. Rotllan N, Chamorro-Jorganes A, Araldi E, Wanschel AC, Aryal B, Aranda JF, Goedeke L, Salerno AG, Ramírez CM, Sessa WC, et al: Hematopoietic Akt2 deficiency attenuates the progression of atherosclerosis. FASEB J 29: 597-610, 2015.

27. Li Y, Yan L, Zhang W, Hu N, Chen W, Wang H, Kang M and $\mathrm{Ou} \mathrm{H}$ : MicroRNA-21 inhibits platelet-derived growth factor-induced human aortic vascular smooth muscle cell proliferation and migration through targeting activator protein-1. Am J Transl Res 6: 507-516, 2014.

28. Li P, Liu Y, Yi B, Wang G, You X, Zhao X, Summer R, Qin Y and Sun J: MicroRNA-638 is highly expressed in human vascular smooth muscle cells and inhibits PDGF-BB-induced cell proliferation and migration through targeting orphan nuclear receptor NOR1. Cardiovasc Res 99: 185-193, 2013.

29. Yang J, Chen L, Ding J, Fan Z, Li S, Wu H, Zhang J, Yang C, Wang $\mathrm{H}$, Zeng $\mathrm{P}$ and Yang J: MicroRNA-24 inhibits high glucose-induced vascular smooth muscle cell proliferation and migration by targeting HMGB1. Gene 586: 268-273, 2016.

30. Tao ZH, Wan JL, Zeng LY, Xie L, Sun HC, Qin LX, Wang L, Zhou J, Ren ZG, Li YX, et al: miR-612 suppresses the invasive-metastatic cascade in hepatocellular carcinoma. J Exp Med 210: 789-803, 2013.

This work is licensed under a Creative Commons Attribution-NonCommercial-NoDerivatives 4.0 International (CC BY-NC-ND 4.0) License. 